\title{
A Comparison of Metabolic Disruptions in Alzheimer Disease and Parkinson Disease
}

\author{
Cade Plyer $^{1}$, Fen-Lei Chang ${ }^{2}$ \\ ${ }^{1,2}$ Indiana University School of Medicine; ${ }^{2}$ Parkview Health, Department of Neurology
}

\section{Background and Hypothesis:}

There has been a shift in focus toward metabolic factors in the study of neurodegenerative disorders as their importance has been highlighted by recent discoveries involving disruption of shared mechanisms. Alzheimer disease (AD) and Parkinson disease (PD) share similar metabolic risk factors. We hypothesize that type 2 diabetes mellitus (T2DM) will be most associated with $A D$, while gastrointestinal (GI) problems will be most associated with PD; however, both $A D$ and $P D$ will have greater associations with $\mathrm{GI}$ problems and T2DM relative to control.

\section{Project Methods:}

This retrospective chart review included three groups: AD, PD, and control. All data were obtained from neurology clinics in Parkview Health's electronic medical record (EMR). ICD-10 codes identified 65+ year old patients who had visited a clinic within the last year and had a diagnosis of either AD, PD, or non-neurodegenerative symptoms (control): neuropathy, headaches, or seizures. ANOVA and chi-square tests were performed for group analyses, and multiple regression analyses were performed between $A D$ and PD disease severity and various metabolic predictors (e.g., HbA1c, total cholesterol).

\section{Results:}

This study currently consists of a protocol. Data collection and analysis will take place between May 2021 and August 2022.

\section{Potential Impact:}

This study aims to fully characterize certain metabolic disruptions with AD and PD by analyzing a ten-year span of clinical data in the same patient population; this may reveal risk factors that are amenable to clinical interventions. This may also facilitate the understanding of Lewy body dementia, a disorder that contains elements of both AD and PD. The main metabolic elements explored in this study are those concerning GI problems (e.g., constipation) and T2DM (e.g., elevated $\mathrm{HbA} 1 \mathrm{c})$. These associations will form the basis of a follow-up study that investigates the potential mechanisms linking these disruptions with pathogenesis of either AD or PD. 